\title{
Neutrophil-lymphocyte ratio in catatonia
}

\section{Sengul Kocamer SAHin ${ }^{1}$}

https://orcid.org/0000-0002-5371-3907

\author{
Celal Yaşamali ${ }^{1}$ \\ https://orcid.org/0000-0002-2813-2270 \\ MuHAMMET BERKAY ÖZYÜREK² \\ https://orcid.org/0000-0002-7016-1411
}

GÜlçıN ELBOĞA ${ }^{1}$

https://orcid.org/0000-0003-3903-1835

Abdurrahman AltindaĞ ${ }^{1}$

https://orcid.org/0000-0001-5531-4419

AHMEt ZiYa ŞAHIN 3

https://orcid.org/0000-0001-5853-8709

I Department of Psychiatry, Faculty of Medicine, Gaziantep University, Gaziantep, Turkey.

${ }^{2}$ Department of Psychiatry, Faculty of Medicine, Balıkesir University, Balıkesir, Turkey.

${ }^{3}$ Department of Internal Medicine, Faculty of Medicine, Sanko University, Gaziantep, Turkey.

Received: 05/18/2019 - Accepted: 01/14/2020

DOl: 10.1590/0101-60830000000232

\begin{abstract}
Background: There is growing evidence of subclinical inflammation in mental disorders. Objective: The aim of this study was to investigate frequency of symptoms of catatonia and the newly diagnosed subclinical inflammatory markers which are neutrophil/lymphocyte (NLR), platelet/lymphocyte (PLR), monocyte/lymphocyte (MLR) ratios in catatonia patients due to mental disorders. Methods: Patients who were admitted to psychiatry clinic with the diagnosis of catatonia according to DSM 5 in the last two years and equal number of control group were included in this retrospective study. Univariate analysis of covariance controlled for possible confounders was used to compare NLR, PLR, MLR ratios between patients and the control group. Results: A total of 34 catatonia patients and 34 healthy controls were included in the study. Patients' mean age was $30.88+13.4$. NLR value was significantly higher in the patient group than control group. There was no significant difference between the patients and control group according to PLR, MLR values. Discussion: The presence of subclinical inflammation in catatonic syndrome due to mental disorders should be considered. Subclinical inflammation that was observed in numerous mental disorders continues in catatonia due to mental disorders. Large-scale studies are needed to determine the role of inflammation in catatonia.
\end{abstract}

Sahin SK et al. / Arch Clin Psychiatry. 2020;47(2):55-8

Keywords: Catatonia, inflammation, neutrophil-lymphocyte ratio, platelet lymphocyte ratio, monocyte/lymphocyte ratio, symptom frequency.

\section{Introduction}

Catatonia, first described by Karl Kahlbaum, is a neuropsychiatric disorder characterized by the presence of motor, behavioral, emotional and vegetative symptoms ${ }^{1}$. DSM-IV included the schizophrenia catatonic subtype. Catatonic type: a type of schizophrenia in which the clinical picture is dominated by at least two of the following: 1) motoric immobility as evidenced by catalepsy (including waxy flexibility) or stupor; 2) excessive motor activity (that is apparently purposeless and not influenced by external stimuli); 3) extreme negativism (an apparently motiveless resistance to all instructions or maintenance of a rigid posture against attempts to be moved) or mutism; 4) peculiarities of voluntary movement as evidenced by posturing (voluntary assumption of inappropriate or bizarre postures), stereotyped movements, prominent mannerisms, or prominent grimacing; 5) echolalia or echopraxia. In DSM-5 catatonia is defined by the presence of three or more of the following symptoms; stupor, catalepsy, waxy flexibility, mutism, negativism, posturing, mannerism, stereotypes, agitation, grimacing, echolalia, echopraxia ${ }^{2}$. The most common catatonic symptoms are staring, mutism, negativism, stupor, withdrawal, rigidity, posturing and catalepsy3,4. It can be seen as neurological disorders, endocrine, metabolic disorders, acute onset due to systemic infections and also psychiatric conditions $s^{5}$ and it is increasingly becoming one of the main psychopathological areas within the spectrum of schizophrenia and other psychotic disorders and mood disorders 6 .

It has been thought that there is central GABAergic, dopaminergic and glutamatergic dysfunction in physiopathology of catatonia?
Its pathophysiology is not clearly understood ${ }^{7}$. Medical catatonia is related to inflammation, neural damage, neurodevelopmental disorders, structural brain pathologies that affect central nervous system $^{8}$. Researches on etiological basis of catatonia due to psychiatric disorders are still insufficient. Various evidence has been put forward regarding familial-genetic factors ${ }^{9}$.

Subclinical inflammation is defined as a low grade inflammatory which may continue in symptom free periods of inflamatuar diseases ${ }^{10-12}$. High-sensitive C-reactive protein (hs-CRP), interleukin 6 (IL-6), IL-1 receptor antagonist are some of the subclinical inflammatory markers which were used in clinical studies ${ }^{13-15}$. Subclinical inflammation has been described in also mental disorders like major depressive disorder, bipolar disorder, schizophrenia and schizoaffective disorder which can be presented with catatonia ${ }^{15-17}$. Recent studies support this inflammation hypothesis in severe mental disorders ${ }^{18}$. In fact, the evidence of subclinical inflammation presence in mental disorders that do not exhibit catatonic features is also prominent ${ }^{19-21}$. However, the role of inflammation in mental disorders is not clearly understood. Also there are studies suggest that auto-immune diseases increase the risk of schizophrenia and bipolar disorders ${ }^{21,22}$. It has been reported that systemic lupus erythematous which is a chronic inflammatory auto-immune disease presented with catatonia symptoms and was improved with lupus treatment ${ }^{23}$.

One of the determinants of chronic inflammation is the white blood cell count and its subtypes. Neutrophil/lymphocyte ratio (NLR) is a new parameter indicating the subclinical inflammation ${ }^{24,25}$. Recently, platelet/lymphocyte (PLR), monocyte/lymphocyte (MLR) ratio has been used to determine inflammation ${ }^{26,27}$. 
In this study, we aimed to explore the frequency of symptoms of catatonia in primary outcome and investigate whether there is an increase in NLR, PLR, and MLR in catatonia patients as part of mental disorders that have been implicated with subclinical inflammation as secondary outcome.

\section{Methods}

Patients diagnosed with catatonia according to the DSM-5 criteria and treated in the psychiatry clinic were included in this retrospective study between November 2016 and November 2018. The approval for the study was obtained from the Ethics Committee before collection of data. Totally 43 patients were detected in this review of inpatients records. Patients with severe neurological disease, diabetes mellitus and other endocrinopathies, patients with liver disease, malignant disease, mental retardation, obesity (BMI $\geq 30$ ), pregnancy, using anti-inflammatory drugs (NSAIDs, corticosteroids) and alcohol/ substance use disorder or addiction history were excluded from the study. Rest of the patients (34) were included the study. Symptoms of the patients according to DSM 5 criteria were examined. Magnetic resonance imaging without contrast had been performed in all patients and no organic brain damage had been observed. Patients' hemoglobin values had been within normal range. Preliminary evaluation of all patients had been completed and patients had not had any active infections. Patients' thyroid stimulant hormone values had been within normal range. No substance had been detected in the urine. The control group was chosen as the last 34 healthy individuals without any disabilities who applied to the health board in the last 6 months.

Sociodemographic and clinical variables as age, gender, education, smoking status, weight and length had been recorded. Weight $/$ length $2\left(\mathrm{~kg} / \mathrm{m}^{2}\right)$ formula was used to calculate BMI.

NLR, PLR, MLR which are subclinical inflammatory markers were compared between 34 patients and 34 control groups. Descriptive statistics were used for the demographic characteristics of 34 patients with catatonia. $\chi^{2}$ test was used to compare categorical variables. t-test was used for comparison of normally distributed variables between the two groups. Mann-Whitney $U$ test was used for comparison of abnormal distributed variables. Finally, the effects of factors such as age, sex, smoking and BMI on NLR, PLR, MLR levels of patients were analyzed using univariate analysis of covariance (ANCOVA). SPSS 22.0 (IBM Corporation, Armonk, New York, United States) software was used in the analysis of variables.

\section{Results}

A total of 68 cases (34 catatonia and 34 healthy controls) were included in our study. Eighteen patients $(53 \%)$ were female, 16 were male $(47 \%)$. Mean age was $30.88+13.4$; mean education years were $6.08+3.2$. Seven of the catatonia patients had no psychiatric diagnosis in the past. Of these 7 patients who were admitted for catatonia for the first time, 3 were diagnosed with bipolar manic episode and 4 with major depression at the end of the treatment in psychiatry clinic. Of the 27 patients who had psychiatric follow-up in the past, 5 had schizophrenia, 14 had bipolar disorder ( 9 had manic episodes before catatonia, 5 had depressive episodes) and 8 had major depression (4 with psychotic features). There were no significant differences between the groups in terms of age, sex, smoking and marital status (Table 1).

The most common symptoms in patients were mutism $70.5 \%$ and negativism $64.7 \%$. The symptoms and frequencies according to DSM 5 are shown in Table 2.

NLR ratio was significantly higher in patients with catatonia compared to controls $(\mathrm{p}=0.004)$. No difference was found between the patient and the control group in terms of MLR, PLR (Table 3).

Covariance analysis revealed that NLR levels were significantly higher in patients compared to control group (Table 4).
Table 1. Sociodemogrophic data

\begin{tabular}{|l|c|c|c|c|}
\hline \multicolumn{2}{|c|}{} & \multicolumn{2}{c|}{ Case control } & \multirow{2}{*}{ P } \\
\cline { 3 - 4 } \multicolumn{2}{|c|}{ Sex } & Catatonia & Control & \\
\cline { 2 - 4 } & Female & $16(47 \%)$ & $13(38.2 \%)$ & \multirow{2}{*}{0.469} \\
\hline Age & & $30.88 \pm 13.4$ & $36,4 \pm 12,2$ & 0.084 \\
\hline Education (year) & & $6.08 \pm 3.2$ & $7,58 \pm 3,7$ & 0.086 \\
\hline Body mass index & & $23.5 \pm 7.5$ & $22.9 \pm 1.26$ & 0.054 \\
\hline \multirow{2}{*}{ Smoke } & Yes & 14 & 11 & \multirow{2}{*}{0.458} \\
\cline { 2 - 4 } & No & 20 & 23 & \\
\hline \multirow{2}{*}{ Marital Status } & Married & 20 & 26 & \multirow{2}{*}{0.097} \\
\cline { 2 - 4 } & Single & 14 & 8 & \\
\hline
\end{tabular}

Table 2. Frequency of catatonia symptoms according to DSM 5 criteria

\begin{tabular}{|l|c|}
\hline Catatonia symptoms & Symptom frequency \\
\hline Mutism & $24(70.5 \%)$ \\
\hline Negativism & $22(64.7 \%)$ \\
\hline Waxy Flexibility & $18(51.4 \%)$ \\
\hline Posturing & $14(41.7 \%)$ \\
\hline Stupor & $12(35.2 \%)$ \\
\hline Catalepsy & $9(26.4 \%)$ \\
\hline Stereotypes & $8(22.8 \%)$ \\
\hline Agitation & $8(22.8 \%)$ \\
\hline Grimacing & $6(17.6 \%)$ \\
\hline Mannerism & $3(8.8 \%)$ \\
\hline Echolalia & $1(2.9 \%)$ \\
\hline Echopraxia & 0 \\
\hline
\end{tabular}

Table 3. Comparison of NLR, PLR, MLR of patients and control group

\begin{tabular}{|l|c|c|c|}
\hline \multirow{2}{*}{} & \multicolumn{2}{|c|}{ Mean \pm standard deviation } & \multirow{2}{*}{$P$} \\
\cline { 2 - 3 } & Catatonia & Control & \\
\hline NLR & $2.81 \pm 1.5$ & $1.95 \pm 0.70$ & $0.004^{*}$ \\
\hline MLR & $0.33 \pm 0.1$ & $0.27 \pm 0.1$ & 0.080 \\
\hline PLR & $134.0 \pm 47.2$ & $147.1 \pm 52.6$ & 0.287 \\
\hline
\end{tabular}

${ }^{*} \mathrm{p}<0.05$

Table 4. Results of NLR, PLR, MLR levels between patients and control group with ANCOVA

\begin{tabular}{|l|c|c|c|}
\hline \multirow{2}{*}{} & \multicolumn{2}{|c|}{ Mean \pm standard error } & \multirow{2}{*}{ P } \\
\cline { 2 - 3 } & Catatonia & Control & \\
\hline NLR & $2.85 \pm 0.20$ & $2.09 \pm 0.21$ & $0.010^{*}$ \\
\hline MLR & $0.33 \pm 0.02$ & $0.29 \pm 0.02$ & 0.298 \\
\hline PLR & $133.8 \pm 8.6$ & $154.7 \pm 8.9$ & 0.100 \\
\hline
\end{tabular}

${ }^{*} \mathrm{p}<0.05$

\section{Discussion}

In this study, we investigated NLR, PLR, and MLR in catatonia patients as part of mental disorders that have been implicated with subclinical inflammation. The results of our study put forth that there is a subclinical inflammation (higher NLR) in catatonia patients due to mental disorders. Additionally, frequency of clinical features related with catatonia will be discussed.

The first finding of our study is the frequency of mood disorders in catatonia patients. Catatonia is associated with many pathophysiological processes and is most commonly associated with mood disorders ${ }^{28}$. In our study, 29/34 (85\%) of the patients were followed up with mood disorder and 17/34 (50\%) with bipolar disorder.

Excitation (72.7\%), immobility/stupor (21.4\%) and mutism (15.6\%) were reported as the most common symptoms in catatonia due to medical conditions ${ }^{28}$. We found that mutism $(70.5 \%)$ and 
negativism $(64.7 \%)$ were the most common symptoms in catatonia due to mental disorders compatible with previous studies ${ }^{3,4}$.

Considering the dysfunction associated with GABAergic, dopaminergic, and glutamatergic neurotransmitter ${ }^{7}$ in the mechanism of catatonia, the link between neurotransmitters and inflammation is noteworthy. Pro-inflammatory cytokines interact with the cytokine network in the central nervous system; neurotransmitter metabolism, neuroendocrine function, synaptic plasticity and related motor activity so these inflammatory cytokines can affect almost every aspect of brain function related to motivational behavior ${ }^{29,30}$. These effects of the immune system in the brain can cause behavioral consequences and neuropsychiatric disorders ${ }^{29,30}$. Large-scale studies are needed to determine the role of inflammation in catatonic patients and to evaluate NLR separately bipolar disorder, major depression and psychosis with catatonia.

According to our literature review, this study is the first study investigating systemic low grade inflammation in catatonia syndrome due to psychiatric disorders. There are evidence that proinflammatory cytokines, CRP, oxidative parameters increase in psychiatric disorders. These are findings that support subclinical inflammation ${ }^{16,17,31,32}$. Recent studies have explored the subclinical inflammation in severe mental disorders using NLR, MLR, and PLR. A study found that NLR was higher in patients with drug naïve major depressive disorder patients ${ }^{33}$ and NLR and PLR were found to be higher in bipolar disorder both depressive and euthymic phase ${ }^{33,34}$. A study compared bipolar disorder and schizophrenia in terms of subclinical inflammation and found that NLR, PLR, MLR higher in patients with bipolar disorder or schizophrenia than in control group, NLR, MLR but not PLR were higher in patients with schizophrenia than in patients with bipolar disorder ${ }^{27}$. Another study was found that NLR, MLR but not PLR were higher in patients with bipolar disorder in manic state. A recent meta-analysis identified 11 studies exploring the NLR, PLR, MLR in mood disorders has shown that NLR and PLR higher in patients with bipolar disorders than healthy controls while the differences were not significant in subgroup analysis among studies including only patients with bipolar disorder euthymic phase ${ }^{35}$. Another recent meta-analysis identified 11 studies exploring NLR, PLR, MLR in non-affective psychosis has shown that NLR an d MLR higher in patients than control group ${ }^{36}$. So predominantly NLR seems to be more reliable inflammatory markers in severe mental disorders ${ }^{18}$.

PLR was not shown significant differences between patients and controls in this study. But, NLR was found to be more reliable marker for subclinical inflammation than $\mathrm{PLR}^{37}$. So these results support the presence of subclinical inflammation in catatonia.

NLR is low cost and reproducible tests that can be easily determined, calculated in simple laboratory conditions. NLR has been shown to predict poor prognosis and major inflammation in chronic medical disorders ${ }^{38,39}$. The result of our study supports the presence of subclinical inflammation in catatonia. In our literature review, we could not find a study of systemic inflammation in catatonia patients. However, myelin abnormalities have been described to cause low grade neuroinflammation and catatonic behavior ${ }^{38}$. Probably myelin abnormalities and systemic low grade inflammation are interrelated. The underlying mechanism of neuroinflammation caused catatonic behavior is not fully understood ${ }^{38}$.

The retrospective design of the study and the low number of patients are limitations of this study. The drug use duration of the patients was unknown. Patient group was not a homogenous group in terms of mental disorders. Seven of them had no psychiatric diagnosis in the past and they were newly diagnosed. Comparisons are needed in the same mental diagnostic groups. Also NLR, MLR, PLR rates may be a weak parameter in the determination of inflammation and it is recommended that they should be supported through studies using other inflammatory markers together. There was a statistical trend for age and education between groups. The diagnosis of catatonia was made according to the DSM-5, and a clinical examination was performed by two psychiatrists. But, structured psychiatric assessments, such as the SCID II were not performed.
As a result, the presence of a subclinical inflammation in the catatonic syndrome due to mental disorder should be considered in addition to medical and neurological conditions. NLR as a subclinical inflammatory marker is higher in catatonia. Subclinical inflammation that was observed in numerous mental disorders continues in catatonia due to mental disorders. NLR may be a more reliable marker for subclinical inflammation in severe mental disorders. This study will guide the future studies in the evaluation of the etiology of catatonia and evaluation of treatment options.

\section{Acknowledgements}

The authors acknowledge to Assoc. Prof. Dogan I. for assistance with statistical analysis.

\section{Disclosure}

The authors report no conflict of interests.

\section{References}

1. Walther S, Strik W. Catatonia. CNS Spectr. 2016;21(4):341-8.

2. Luchini F, Bartolommei N, Benvenuti A, Mauri M, Lattanzi L. Catatonia from the first descriptions to DSM 5. J Psychopathol. 2015;21:145-51.

3. Dutt A, Grover S, Chakrabarti S, Avasthi A, Kumar S. Phenomenology and treatment of Catatonia: A descriptive study from north India. Indian J Psychiatry. 2011;53(1):36-40.

4. Swain SP, Behura SS, Dash MK. The phenomenology and treatment response in catatonia: a hospital based descriptive study. Indian J Psychol Med. 2017;39(3):323-9.

5. Fink M, Fricchione G, Rummans T, Shorter E. Catatonia is a systemic medical syndrome. Acta Psychiatr Scand. 2016;133(3):250-1.

6. Ungvari GS, Caroff SN, Gerevich J. The catatonia conundrum: evidence of psychomotor phenomena as a symptom dimension in psychotic disorders. Schizophr Bull. 2009;36(2):231-8.

7. Pot AL, Lejoyeux M. La catatonie. L'Encéphale. 2015;41(3):274-9.

8. Oldham MA. The probability that catatonia in the hospital has a medical cause and the relative proportions of its causes: a systematic review. Psychosomatics. 2018;59(4):333-40.

9. Peralta V, Fañanás L, Martín-Reyes M, Cuesta MJ. Dissecting the catatonia phenotype in psychotic and mood disorders on the basis of familial-genetic factors. Schizophr Res. 2018;200:20-5.

10. Ahsen A, Ulu MS, Yuksel S, Demir K, Uysal M, Erdogan M, et al. As a new inflammatory marker for familial Mediterranean fever: neutrophil-to-lymphocyte ratio. Inflammation. 2013;36(6):1357-62.

11. Festa A, D'Agostino Jr R, Howard G, Mykkanen L, Tracy RP, Haffner SM. Chronic subclinical inflammation as part of the insulin resistance syndrome: the Insulin Resistance Atherosclerosis Study (IRAS). Circulation. 2000;102(1):42-7.

12. Ng QX, Soh AYS, Loke W, Lim DY, Yeo WS. The role of inflammation in irritable bowel syndrome (IBS). J Inflamm Res. 2018;11:345-9.

13. Herder C, Hermanns N. Subclinical inflammation and depressive symptoms in patients with type 1 and type 2 diabetes. Semin Immunopathol. 2019;41(4):477-89.

14. Kopp H, Krzyzanowska K, Möhlig M, Spranger J, Pfeiffer A, Schernthaner G. Effects of marked weight loss on plasma levels of adiponectin, markers of chronic subclinical inflammation and insulin resistance in morbidly obese women. Int J Obes (Lond). 2005;29(7):766-71.

15. Elboga G, Sahin SK, Sahin AZ, Altindag A. Serum levels of inflammatory biomarkers in schizoaffective disorders. Acta Med Mediterr. 2017;33(5):863-8.

16. Rosenblat JD, McIntyre RS. Bipolar disorder and inflammation. Psychiatr Clin. 2016;39(1):125-37.

17. Kohler O, Krogh J, Mors O, Eriksen Benros M. Inflammation in depression and the potential for anti-inflammatory treatment. Curr Neuropharmacol. 2016;14(7):732-42.

18. Rodríguez JO, Fernández JD, Mira NO, López-Briz E, Gómez-Pajares F. Neutrophil/lymphocyte ratio: can a non-specific marker of inflammation helps to confirm the inflammatory hypothesis of the serious mental diseases? A case-control study. Med Hypotheses. 2019;130:109279. 
19. Michopoulos V, Powers A, Gillespie CF, Ressler KJ, Jovanovic T. Inflammation in fear- and anxiety-based disorders: PTSD, GAD, and beyond. Neuropsychopharmacology. 2017;42(1):254-70.

20. Dalton B, Bartholdy S, Robinson L, Solmi M, Ibrahim MA, Breen G, et al. A meta-analysis of cytokine concentrations in eating disorders. J Psychiatr Res. 2018;103:252-64.

21. Benros ME, Nielsen PR, Nordentoft M, Eaton WW, Dalton SO, Mortensen PB. Autoimmune diseases and severe infections as risk factors for schizophrenia: a 30-year population-based register study. Am J Psychiatry. 2011;168(12):1303-10.

22. Cremaschi L, Kardell M, Johansson V, Isgren A, Sellgren CM, Altamura AC, et al. Prevalences of autoimmune diseases in schizophrenia, bipolar I and II disorder, and controls. Psychiatry Res. 2017;258:9-14.

23. Pustilnik S, Trutia A. Catatonia as the presenting symptom in systemic lupus erythematosus. J Psychiatr Pract. 2011;17(3):217-21.

24. Kul S, Akyüz AR. Xanthelasma Palpebrarum is Associated with Higher Levels of Neutrophil to Lymphocyte and Platelet to Lymphocyte Ratio. Sakarya Tip Dergisi. 2018;8(1):63-9.

25. Sunbul M, Gerin F, Durmus E, Kivrak T, Sari I, Tigen K, et al. Neutrophil to lymphocyte and platelet to lymphocyte ratio in patients with dipper versus non-dipper hypertension. Clin Exp Hypertens. 2014;36(4):217-21.

26. Cummings M, Merone L, Keeble C, Burland L, Grzelinski M, Sutton K, et al. Preoperative neutrophil: lymphocyte and platelet: lymphocyte ratios predict endometrial cancer survival. Br J Cancer. 2015;113(2):311-20.

27. Özdin S, Sarisoy G, Böke Ö. A comparison of the neutrophil-lymphocyte, platelet-lymphocyte and monocyte-lymphocyte ratios in schizophrenia and bipolar disorder patients - a retrospective file review. Nord J Psychiatry. 2017;71(7):509-12.

28. Denysenko L, Freudenreich O, Philbrick K, Penders T, Zimbrean P, Nejad $S$, et al. Catatonia in Medically Ill Patients: An Evidence-Based Medicine (EBM) Monograph for Psychosomatic Medicine Practice. Nuremberg: The European Association of Psychosomatic Medicine; 2015.
29. Capuron L, Miller AH. Immune system to brain signaling: neuropsychopharmacological implications. Pharmacol Ther. 2011;130(2):226-38.

30. Janova H, Arinrad S, Balmuth E, Mitjans M, Hertel J, Habes M, et al. Microglia ablation alleviates myelin-associated catatonic signs in mice. J Clin Invest. 2018;128(2):734-45.

31. Na KS, Jung HY, Kim YK. The role of pro-inflammatory cytokines in the neuroinflammation and neurogenesis of schizophrenia. Prog Neuropsychopharmacol Biol Psychiatry. 2014;48:277-86.

32. Şahin Ş, Aybastı Ö, Elboğa G, Altındağ A, Tamam L. Major depresyonda elektrokonvulsif terapinin oksidatif metabolizma üzerine etkisi. Cukurova Medical Journal. 2017;42(3):513-7.

33. Demir S, Atli A, Bulut M, İbiloğlu AO, Güneş M, Kaya MC, et al Neutrophil-lymphocyte ratio in patients with major depressive disorder undergoing no pharmacological therapy. Neuropsychiatr Dis Treat. 2015;11:2253-8.

34. Kalelioglu T, Akkus M, Karamustafalioglu N, Genc A, Genc ES, Cansiz A, et al. Neutrophil-lymphocyte and platelet-lymphocyte ratios as inflammation markers for bipolar disorder. Psychiatry Res. 2015;228(3):925-7.

35. Mazza MG, Lucchi S, Tringali AGM, Rossetti A, Botti ER, Clerici M. Neutrophil/lymphocyte ratio and platelet/lymphocyte ratio in mood disorders: a meta-analysis. Prog Neuropsychopharmacol Biol Psychiatry. 2018;84(Pt A):229-36.

36. Mazza MG, Lucchi S, Rossetti A, Clerici M. Neutrophil-lymphocyte ratio, monocyte-lymphocyte ratio and platelet-lymphocyte ratio in non-affective psychosis: A meta-analysis and systematic review. World J Biol Psychiatry. 2019:1-13.

37. Özer S, Yılmaz R, Sönmezgöz E, Karaaslan E, Taşkın S, Bütün İ, et al. Simple markers for subclinical inflammation in patients with Familial Mediterranean Fever. Med Sci Monit. 2015;21:298-303.

38. Dimsdale JE, Dantzer R. A biological substrate for somatoform disorders: importance of pathophysiology. Psychosom Med. 2007;69(9):850-4.

39. Afari ME, Bhat T. Neutrophil to lymphocyte ratio (NLR) and cardiovascular diseases: an update. Expert Rev Cardiovasc Ther. 2016;14(5):573-7. 